\title{
Method of Fault Diagnosis in Gyrocompass Based on CBR
}

\author{
Yundong HAN, Pengshan DU, Changqing YANG, Hongcan HU, Qian HUANG \\ Department of Navigation of Dalian Naval Academy, Dalian, Liaoning Province, China \\ gyro-han@163.com
}

\begin{abstract}
Keywords: ship, naval engineering; fault diagnosis; case-based reasoning; navigation equipment; gyrocompass

Abstract. For the disadvantage of traditional expert system and the characteristic of gyrocompass, a normal process of case-based reasoning (CBR) is analyzed. The advantages of getting knowledge easily, translating clearly and understanding facilely are indicated. Moreover, a method of fault diagnosis of gyrocompass by CBR is proposed and a Fault Diagnosis System based on CBR is presented. The Principle of CBR, design of structure, organization of case, working flow, searching strategy and realization method of this system are elaborated in details. The result of analysis case shows that the correctness and efficiency in fault diagnosis of gyrocompass are improved by the system.
\end{abstract}

\section{Introduction}

Gyrocompass was one of the important navigation equipment, which was of great significance to maintain the good performance of the ship combat readiness. Once a fault occurred, it would make related equipment and system of the ship could not work properly because of no precise course information. Therefore, the gyrocompass fault rapid diagnosis technology was an important research focus. At present, a lot of method of expert system based on fault tree were used on gyrocompass fault diagnosis system, but the method had certain limitations[1-5]. The based on CBR (case-based reasoning (CBR) technology would applied in fault diagnosis of electric compass, to solve the problems of traditional expert system, which included knowledge acquisition and knowledge learning, to improve the accuracy and efficiency of the gyrocompass fault diagnosis.

\section{The general process of CBR technology and main advantages}

The inherent problems existing in the application of expert system method of gyrocompass fault diagnosis system, and it would be solved by using the CBR technology [6-8].

The general process of CBR technology.

CBR was a kind of reasoning method which simulate human analogy thinking, and its reasoning process often had some characteristics of human experience reasoning. The basic process of CBR was: when faced a new problem, the system would retrieved according to the key features in the original case, to discover a candidate case which was the most similar to the seeking problem. Reuse the solution of the candidate case; If not satisfied with the solution, it could be modified to suit for seeking problems; Finally, the modified case save as a new case into the case base, so that it could be a reference when the next time encountered the similar problems. CBR used case as knowledge element, the knowledge acquisition and representation was natural and directly, and it had good self-learning ability, its essence was based on the similarity analogical reasoning, which was keeping with the logic of human analogical thinking.

\section{The main advantage of CBR technology}

The traditional rule-based expert system diagnosis method had shortcomings as follows: knowledge acquisition was difficult; System felt helpless when solved beyond the scope of the problem; The diagnosis reasoning process were abstract, not easy to understand etc [2, 5]. While CBR was reasoning by using the experience that solved the similar problems, retrieved on-site fault cases, could reduce the necessary of acquiring knowledge from experts, and the establishment of the new case was easy; It could give similar solution between the faults and the cases of the diagnosed object did not fully 
matched; The result of diagnosed was a specific case, more lively and vivid, could reflect the fault overview, and easy to understand. Therefore, the technology of CBR could provide a new way to the fault diagnosis of instruments, such as gyrocompass, especially it had more reference value for personnel who lack of diagnostic experience.

\section{Design principle and construction method}

The technology of CBR was used in gyrocompass fault diagnosis, needed to complete CBR principle construct, system structure design, case organization and form design, retrieval strategy and workflow design.

\section{The principle of CBR build}

The principle of CBR problem solving process could be summarized as R4, which including case retrieval (Retrieval), case reuse (Reuse), case revise (Revise) and case to retain(Retain). According to the principle of CBR, its build in gyrocompass fault diagnosis as follows (see figure 1).

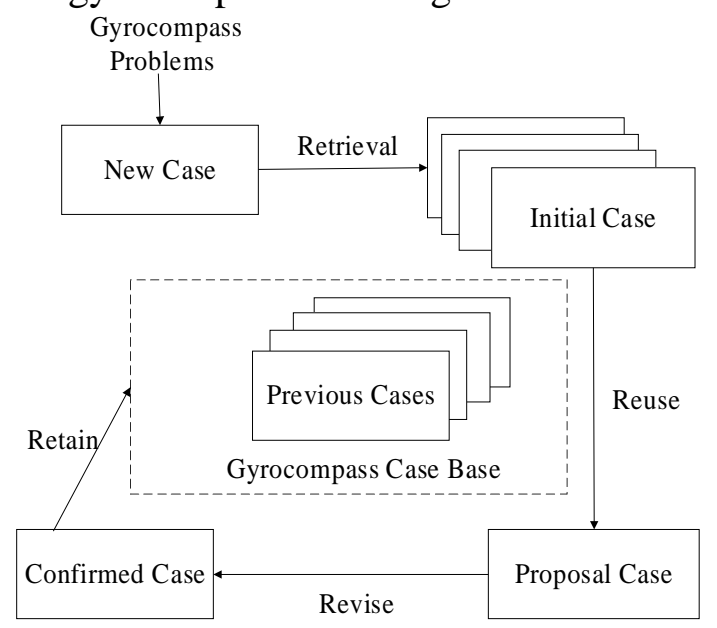

Fig. 1 Schematic of CBR

Because of the CBR retrieved several past gyrocompass cases, and reused by certain way, got the proposal case by correction, save the experience gained, so every time a CBR cycle, was not only the problem solving process, but also the case study process, and the knowledge of the system update process. So through solved problem constantly, and built the new case, to enrich the knowledge of expert system constantly and enlarge the coverage of knowledge, at the same time, evaluated and analyzed of the actual effect by comparison, to improve the accuracy and efficiency of the solution about the gyrocompass fault diagnosis system.

\section{Case organization and Expression of design}

Case organized was in order to say it better, according to the characters of the case and the needs of retrieval, classified and cleared out the case. In the system, the solution of the knowledge unit was used to the specific gyrocompass fault case, its collection made up the system original case base. In the gyrocompass fault diagnosis, a case was a complete gyrocompass fault solution, this solution could be directly used to solve the same or similar fault in the future. Gyrocompass fault diagnosis case had the following features: every fault according to the specific instrument of the fault of the corresponding system, belong to a big kinds of fault cases, such as power box fault belongs to power system fault classes, compass fault belongs to synchronization system fault classes; Each case was a specific example in the fault case class, and the fault case was described by a series of attributes.

In this paper, based on the characteristics of the gyrocompass fault cases, the model of the design was based on these features. The gyrocompass fault had four basic attributes: basic fault information, fault symptom, fault cause and fault measure. The basic fault information was the form of the failure case name and external; The fault symptom was the abnormal symptoms when fault happened; The cause of the fault was the cause that made the fault happened; The fault measures were the concrete measures to solved the fault and the work of the equipment after made the adopt correspond measures. 
The fault in this system was defined as a vector with six dimensions: Case $=\langle\mathrm{I}, \mathrm{F}, \mathrm{S}, \mathrm{K}, \mathrm{M}, \mathrm{P}\rangle, \mathrm{I}$ was the fault case name in the type and the unique identifier about the fault case; $F$ was the fault collection, which was made up with different fault conditions; $\mathrm{S}$ was a sign collection that system displayed when the fault $\mathrm{F}$ occurred; $\mathrm{K}$ was a collection of necessary domain knowledge around the fault F; $\mathrm{M}$ was a cause collection when the fault $\mathrm{F}$ happened; $\mathrm{P}$ was the description and the feedback information about the fault solution.

\section{Case retrieval methods of design}

The retrieval of the gyrocompass case classified two stages about searching for case and matching case symptom, search for the case type was because only the similar cases had the application value, and the same kind of case retrieval was a sign collection of matching [9].

First this system used the classification of the case information to retrieve and determine the type of case, namely the fault instrument belong to which system of the gyrocompass; Then focus on matching the nearest in the same kind of case, discover the most similarity case, at the same time it also could according to the most important feature in the case to retrieve, this could short the retrieval time; After retrieved a set of similar cases, then finally determined which one was the most similar case in the current cases, namely calculated the similarity of two cases. System used k neighbor algorithm with the weighting to match the case. A gyrocompass failure case $\mathrm{T}$ and $\mathrm{S}$, get the similarity according to the following formula. The similarity of case was smaller, showed the gyrocompass failure case $\mathrm{T}$ and $\mathrm{S}$ were closer and the more similar.

$$
\operatorname{Sim}(\mathrm{T}, \mathrm{S})=\sqrt{\frac{\sum_{\mathrm{i}=1}^{\mathrm{n}} \mathrm{W}_{\mathrm{i}}\left(\mathrm{a}_{\mathrm{i}}(\mathrm{T})-\mathrm{a}_{\mathrm{i}}(\mathrm{S})\right)^{2}}{\sum_{\mathrm{i}=1}^{\mathrm{n}} \mathrm{W}_{\mathrm{i}}}}
$$

\section{System work process design}

New case diagnosis module was the core of the gyro fault diagnosis system module and its work process was shown in figure 3.

The user entered new gyrocompass fault symptoms in a new case diagnosis window; First the system would using $\mathrm{k}$ neighbor algorithm to retrieve according to the similarity threshold $\theta$ in the representative case and to see whether the case matching could be achieve; If not, then give the reasons for this failure of matching, represent the case that user input the was a new case, it needed to be learned, then saved the results into the failure case; It would be retrieved from the case base if had similar representative case, then it would use k neighbor algorithm according the similarity threshold $\eta$ to retrieve the most similar instruction case; Then output instruction case, for user and the computer with the interact interface, then let user understand the final diagnosis was referred to which the original case conclusion; After user choose to continue running, the system would according to the case modified the principle, and calculate the final diagnosis; If it cannot diagnosed successful, then return to the failure report, put the failure reasons into further check suggestion and in the form of a failure report back to the user, and then add it into failure case base; If diagnosed successful, output the successful diagnosis report, then add the case into case base.

\section{System implementation and Case analysis}

According to the previous analysis, the high-level programming language was used to develop new gyrocompass fault diagnosis system, and analyzed a case of actual application effect.

Taking a ship to the configuration of a certain type of gyrocompass took place "power box on an ammeter indicates zero" fault as a new case for troubleshooting in one day. According to the work flow system mentioned in the Fig 3, take the sign information input system, get instruction information as shown in TABLE 1.The similarity value was calculated according to the formula (1) mentioned in the Section IV. 
TABLE 1

Instruction information list auto generated by system

Case Number : 0110

Setting Time : D2007-5-12 T09:33

Under System : Power System

Failure Symptom : Side1 : Starting Up Normal With No Abnormalities

Side2 : A Ampere Meter Show 0

in the power box

Side3 : Other Ampere Meter Show

Normal

Fault Cause : Combination Machine Give Out

Three Phase Default Phase

Fault Resource : Side1 : Measure Three Phase, Sure The Default Phase

Side2 : Power Off , Check The

Combination Machine

Side3 : Change Default Phase

Brush

Analysis Result : Similarity was 0.81

According to the instruction that system gave to check the trouble, discovered the combination machine lack of the second phase in the three-phase electricity, power off and checked the combination, discovered that the second phase brush was wear serious, after changed it ruled out the fault successful.

Because this system summed up the more than 120 cases based on collecting more than 380 cases of gyrocompass fault, and put these in the form of case base and knowledge base into the database, thus could ensure these in actual applications, not only match to the fault symptoms of case more accurate and stable, but also gave the instruction information more clear, and easy to understand, at the same time added a new case also would be more convenient and easy.

\section{Conclusion}

In this paper, the CBR technology was applied to the fault diagnosis of gyrocompass, elaborated its principle structure and design method, and improve the shortages of traditional expert system existed effectively. The case analysis result showed that the development of the gyrocompass fault diagnosis system based on CBR could improve the validity and efficient of repair.

\section{References}

[1] Yundong HAN, Qunzhe YUAN, Weiwei YIN, Hairui MA. Research on Principle and Method of Gyrocompass Fault Diagnosis Based on CBR[J]. Navigation of China, 2009,32,(4):22-30

[2] Jiao Wang, Meiling Qi. A Diagnosis Expert System of NC Machine Based on RBR and CBR [J]. Modular Machine Tool \& Automatic Manufacturing Technique, 2011,(1):47-53

[3] Yundong HAN, Yingcai WANG, Yilin YUAN. Research on model about missionoriented equipments maintenance of warship[J]. Journal of Dalian Naval Academy, 2007,(6):33-36

[4] Yongbo HE, Lizhe WANG, Yanhui YANG. Research of CBR in fault diagnosis of aircraft pneumatic system [J]. Modern Manufacturing Engineering, 2013,(3):110-114

[5] Zhinong JIANG, Hui WANG, Zhongqing WEI. Faults Diagnosis Expert System Based on Case

Reasoning and Rule Reasoning [J]. Computer engineering, 2011,37,(1):238-243

[6] Huizhong CHENG. A Study on Weather Radar Fault Diagnosis Expert System Based on Improved Casebased Reasoning [J]. Modern radar, 2013,35,(5):59-62 
[7] Jun YANG, Zhensheng FENG, Kaoli HUANG. Equipped with intelligent fault diagnosis technology [M]. Beijing: National Defense Industry Press, 2006

[8] Ha Manh Tran. Distributed CaseBased Reasoning for Fault Management [J]. Lecture Notes in Computer Science, 2007,4543,(1) ,200-203

[9] Weiyi CHEN, Keming WANG, Wanbin DENG. Research on the case base structure and retrieval based on CBR[J]. Ship Science and Technology 2014,36,(4):126-129 